\title{
On Cycle Related Graphs with Constant Metric Dimension
}

\author{
Murtaza Ali ${ }^{1}$, Gohar $\mathrm{Ali}^{{ }^{*}}$, Usman $\mathrm{Ali}^{2}$, M. T. Rahim ${ }^{1}$ \\ ${ }^{1}$ Department of Mathematics, FAST-National University of Computer and Emerging Sciences, Peshawar, Pakistan \\ ${ }^{2}$ Centre for Advanced Studies in Pure and Applied Mathematics, Bahauddin Zakariya University, Multan, Pakistan \\ Email: *gohar.ali@nu.edu.pk
}

Received September 16, 2011; revised November 2, 2011; accepted December 7, 2011

\begin{abstract}
If $G$ is a connected graph, the distance $d(u, v)$ between two vertices $u, v \in V(G)$ is the length of a shortest path between them. Let $W=\left\{w_{1}, w_{2}, \cdots, w_{k}\right\}$ be an ordered set of vertices of $G$ and let $v$ be a vertex of $G$. The representation $r(v \mid W)$ of $v$ with respect to $W$ is the $k$-tuple $\left(d\left(v, w_{1}\right), d\left(v, w_{2}\right), \cdots, d\left(v, w_{k}\right)\right)$. If distinct vertices of $G$ have distinct representations with respect to $W$, then $W$ is called a resolving set or locating set for $G$. A resolving set of minimum cardinality is called a basis for $G$ and this cardinality is the metric dimension of $G$, denoted by $\operatorname{dim}(G)$. A family $\mathcal{G}$ of connected graphs is a family with constant metric dimension if $\operatorname{dim}(G)$ is finite and does not depend upon the choice of $G$ in $\mathcal{G}$. In this paper, we show that dragon graph denoted by $T_{n, m}$ and the graph obtained from prism denoted by $2 C_{k}+\left\{x_{k} y_{k}\right\}$ have constant metric dimension.
\end{abstract}

Keywords: Metric Dimension; Basis; Resolving Set; Dragon Graph

\section{Notation and Preliminary Results}

If $G$ is a connected graph, the distance $d(u, v)$ between two vertices $u, v \in V(G)$ is the length of a shortest path between them. Let $W=\left\{w_{1}, w_{2}, \cdots, w_{k}\right\}$ be an ordered set of vertices of $G$ and let $v$ be a vertex of $G$. The representation of the $v$ with respect to $W$ is denoted by $r(v \mid W)$ is the $k$-tuple

$\left(d\left(v, w_{1}\right), d\left(v, w_{2}\right), \cdots, d\left(v, w_{k}\right)\right)$. If distinct vertices of $G$ have distinct representations with respect to $W$, then $W$ is called a resolving set or locating set for $G$ [1]. A resolving set of minimum cardinality is called a metric basis for $G$ and its cardinality is the metric dimension of $G$, denoted by $\operatorname{dim}(G)$. The concepts of resolving set and metric basis have previously appeared in the literature (see [1-14]).

For a given ordered set of vertices

$W=\left\{w_{1}, w_{2}, \cdots, w_{k}\right\}$ of a graph $G$, the ith component of $r(v \mid W)$ is $0 \mathrm{f}$ and only if $v=w_{i}$. Thus, to show that $W$ is a resolving set it sufficient to verify that $r(x \mid W) \neq r(y \mid W)$ for each pair of distinct vertices $x, y \in V(G) \backslash W$.

Motivated by the problem of uniquely determining the location of an intruder in a network, the concept of metric dimension was introduced by Slater in [2] and studied independently by Harary et al. [3]. Applications

\footnotetext{
"Corresponding author.
}

of this invariant to the navigation of robots in networks are discussed in [4] and applications to chemistry in [1] while applications to problems of pattern recognition and image processing, some of which involve the use of hierarchical data structures are given in [5].

By denoting $G+H$ the join of $G$ and $H$, a fan is $f_{n}=K_{1}+P_{n}$ for $n \geq 1$ and Jahangir graph $J_{2 n},(n \geq 2)$ (also known as gear graph) is obtained from the wheel $W_{2 n}$ by alternately deleting $n$ spokes. Caceres et al. [6] found the metric dimension of fan $f_{n}$ and Tomescu et al. [7] found the metric dimension of Jahangir graph $J_{2 n}$. Also Tomescu et al. [8] the partition and connected dimension of wheels.

Chartrand et al. proved:

Theorem 1: [1] A graph $G$ has metric dimension 1 if and only if $G$ is a path.

Hence paths on $n$ vertices constitute a family of graphs with constant metric dimension. Similarly, cycles with $n(\geq 3)$ vertices also constitute such a family of graphs as their metric dimension is 2. Since prisms $D_{n}$ are the trivalent plane graphs obtained by the cartesian product of the path $P_{2}$ with a cycle $C_{n}$, hence they constitute a family of 3 -regular graphs with constant metric dimension. Also Javaid et al. proved in [9] that the plane graph antiprism $A_{n}$ constitutes a family of regular graphs with constant metric dimension as $\operatorname{dim}\left(A_{n}\right)=3$ for every $n \geq 5$. 
Let $2 C_{n}+\left\{x_{n} y_{n}\right\}$ be a family of graphs of order $2 n$ obtained from a prism $D_{n}$ as shown in Figure 1 and Figure 2 respectively, by deleting the spokes $x_{i} y_{i}$ for $i \in\{1,2, \cdots, n-1\}$. We prove the following.

Theorem 2: Let $G=2 C_{n}+\left\{x_{n} y_{n}\right\}$ with $|V(G)|=2 n$, then $\operatorname{dim}(G)=2$ for $n \geq 3$.

Let $C_{n}$ be a cycle with vertex set $V\left(C_{n}\right)=\left\{v_{1}, v_{2}, \cdots, v_{n}\right\}$ and $P_{m+1}$ be a path with vertex set $V\left(P_{m+1}\right)=\left\{v_{n}=u_{0}, u_{1}, \cdots, u_{m}\right\}$. Dragon graph $T_{n, m}$ as shown in Figure 3, is a graph of order $n+m$ obtained by identifying $v_{n}$ of $C_{n}$ with $u_{0}$ of $P_{m+1}$. We prove the following.

Theorem 3: For all $n \geq 3, m \geq 2 \operatorname{dim}\left(T_{n, m}\right)=2$.

\section{Proofs}

Proof of the Theorem 2: By Theorem 1, $\operatorname{dim}(G) \geq 2$. We only need to show that $W=\left\{y_{1}, x_{1}\right\}$ is a resolving set for $G$, which is obviously of minimal cardinality.



Figure 1. Prism $D_{n}$.

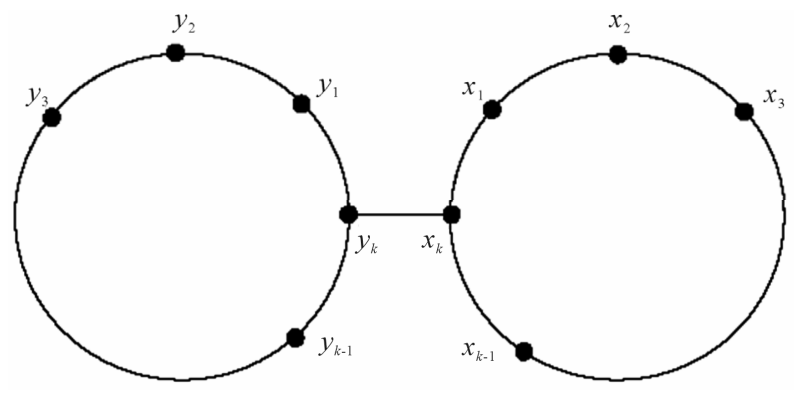

Figure 2. Graph $2 C_{k}+\left\{x_{k} y_{k}\right\}$.

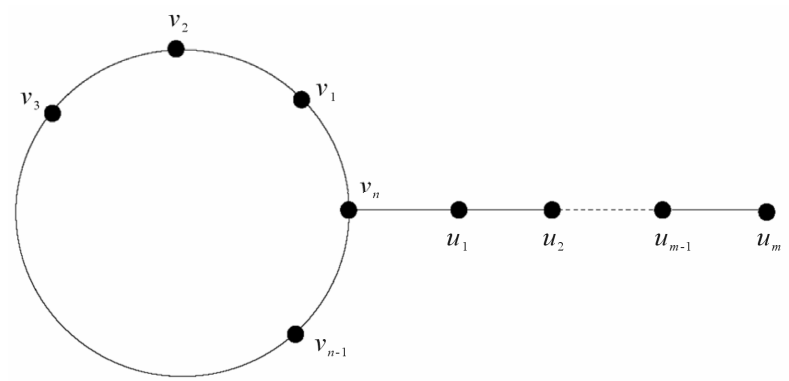

Figure 3. Dragon graph.
Case (a) When $n=2 k$ for $k \in \mathbb{N}$. Representations of all vertices from $V(G) \backslash\left\{y_{1}, x_{1}\right\}$ are as follows,

$$
r\left(x_{i+1} \mid W\right)= \begin{cases}(i, i+3), & 1 \leq i \leq k-1 ; \\ (n-i, n-i+1), & k \leq i \leq n-1 ; \\ (i+3, i), & 1 \leq i \leq k-1 ; \\ (n-i+1, n-i), & k \leq i \leq n-1 .\end{cases}
$$

It is easy to check that all the above representations are distinct. For example, suppose that

$(s+3, s)=(n-j, n-j+1)$ for some fixed $s$ and $j$. Then $s=n-j-3$ and $s=n-j+1$, a contradiction.

Case (b) When $n=2 k+1$ for $k \in \mathbb{N}$. Representations of vertices from $V(G) \backslash\left\{y_{1}, x_{1}\right\}$ are as follows,

$$
r\left(y_{i+1} \mid W\right)= \begin{cases}(i, i+3), & 1 \leq i \leq k-1 ; \\ (k, k+2), & \\ (n-i, n-i+1), & k+1 \leq i \leq n-1 ; \\ (i+3, i), & 1 \leq i \leq k-1 ; \\ (k+2, k), & \\ (n-i+1, n-i), & k+1 \leq i \leq n-1 .\end{cases}
$$

All the above representations are also distinct.

Proof of the Theorem 3: By Theorem 1, $\operatorname{dim}\left(T_{n, m}\right) \geq 2$. We only need to show that there is a resolving set $W$ of cardinality 2 .

Case (a) When $n=2 k$ for $k \in \mathbb{N}$. The set $W=\left\{v_{k}, v_{k+1}\right\}$ is a resolving set for the graph $T_{n, m}$. Representations of all vertices from $V(G) \backslash W$ are as follows,

$$
r\left(v_{i} \mid W\right)= \begin{cases}(k-i, k-i+1), & 1 \leq i \leq k-1 \\ (i-k, i-k-1), & k+2 \leq i \leq n\end{cases}
$$

and

$$
r\left(u_{i} \mid W\right)=(k+i, k+i-1), \quad 1 \leq i \leq m .
$$

It is easy to check that all the representations are distinct. For example, suppose that $(k+s, k+s-1)=(j-k, j-k-1)$ for some fixed $s$ and $j$. Then $j=2 k+s>n$ because $1 \leq s$, a contradiction.

Case (b) When $n=2 k+1$ for $k \in \mathbb{N}$. The set $W=\left\{v_{1}, v_{n-1}\right\}$ is a resolving set for the graph $T_{n, m}$. Representations of all vertices from $V(G) \backslash W$ are as follows,

$$
\begin{aligned}
& r\left(v_{i} \mid W\right)=\left\{\begin{array}{cc}
(i-1, i+1), & 2 \leq i \leq k-1 ; \\
(i-1, n-i-1), & k \leq i \leq k+1 ; \\
(n-i+1, n-i-1), & k+2 \leq i \leq n-2 ;
\end{array}\right. \\
& r\left(v_{n} \mid W\right)=(1,1) ;
\end{aligned}
$$

and

$$
r\left(u_{i} \mid W\right)=(i+1, i+1), \quad 1 \leq i \leq m
$$


All the above representations are distinct.

\section{Acknowledgements}

This research is partially supported by FAST-National University of Computer and Emerging Sciences, Peshawar, Bahauddin Zakariya University, Multan and Higher Education Commission of Pakistan.

\section{REFERENCES}

[1] J. Caceres, C. Hernando, M. Mora, I. M. Pelayo, M. L. Puertas, C. Seara and D. R. Wood, "On the Metric Dimension of Some Families of Graphs," Electronic Notes in Discrete Mathematics, Vol. 22, 2005, pp. 129133.

[2] G. Chartrand, L. Eroh, M. A. Johnson and O. R. Oellermann, "Resolvability in Graphs and Metric Dimension of a Graph,” Discrete Applied Mathematics, Vol. 105, 2000, pp. 99-113. doi:10.1016/S0166-218X(00)00198-0

[3] F. Harary and R. A. Melter, "On the Metric Dimension of a Graph,” Ars Combinatoria, Vol. 2, 1976, pp. 191-195.

[4] I. Javaid, M. T. Rahim and K. Ali, "Families of Regular Graphs with Constant Metric Dimension,” Utilitas Mathematica, Vol. 75, 2008, pp. 21-33.

[5] S. Khuller, B. Raghavachari and A. Rosenfeld, "Localization in Graphs,” Technical Report CS-TR-3326, University of Maryland at College Park, 1994.
[6] R. A. Melter and I. Tomescu, "Metric Bases in Digital Geometry," Computer Vision, Graphics, and Image Processing, Vol. 25, No. 1, 1984, pp. 113-121. doi:10.1016/0734-189X(84)90051-3

[7] P. J. Slater, "Dominating and Reference Sets in Graphs," Journal of Mathematical Physics, Vol. 22, 1998, pp. 445-455.

[8] I. Tomescu and I. Javaid, "On the Metric Dimension of the Jahangir Graph,” Bulletin Mathématique de la Société des Sciences. Mathématiques de Roumanie, Vol. 50, No. 4, 2007, pp. 371-376.

[9] K. Karliraj and V. J. Vernold, "On Equatable Coloring of Helm and Gear Graphs,” International Journal of Mathematical Combinatorics, Vol. 4, No. 1, 2010, pp. 32-37.

[10] I. Javaid, "On the Connected Partition Dimension of Unicyclic Graphs,” Journal of Combinatorial Mathematics and Combinatorial, Vol. 65, 2008, pp. 71-77.

[11] I. Javaid and S. Shokat, "On the Patition Dimension of Some Wheel Related Graph,” Journal of Prime Research in Mathematics, Vol. 4, 2008, pp. 154-164.

[12] M. Ali, M. Imran, A. Q. Baig, M. K. Shafiq and G. Ali, "On the Metric Dimension of Mobius Ladders," Ars Combinatoria, in press.

[13] I. Tomescu, I. Javaid, et al., "On the Patition Dimension and Connected Partition Dimension Wheels," Ars Combinatoria, Vol. 84, 2007, pp. 311-317.

[14] I. Javaid, et al., “Fault-Tolerance in Resolvibility,” Utilitas Mathematica, Vol. 80, 2009, pp. 263-275. 